\title{
Metástasis cutánea de cáncer de colon: una forma de presentación inusual y tardía a tener en cuenta
}

\section{Cutaneous metastasis from colon cancer: an unusual and late presentation form to considerate}

\author{
Jeancarlos J. Trujillo-Díaz*, María Ruiz-Soriano, Mario A. Montes-Manrique, José C. López-López, \\ Andrea Vázquez-Fernández y Juan P. Beltrán de Heredia y Rentería \\ Servicio de Cirugía General y del Aparato Digestivo, Hospital Clínico Universitario, Valladolid, España
}

\begin{abstract}
Resumen
Introducción: El cáncer colorrectal es un gran problema de salud por su alta incidencia y mortalidad. Las metástasis cutáneas son raras, con una incidencia < 5\%. Caso clínico: Varón con antecedente de colectomía por neoplasia de colon que 6 años después presenta una lesión cutánea. La biopsia informa de metástasis colónica, por lo que se realiza resección quirúrgica radical. La anatomía patológica confirma un adenocarcinoma moderadamente diferenciado compatible con metástasis colónica. Discusión y conclusiones: Las metástasis cutáneas son raras y cuando aparecen nos indican una enfermedad avanzada, por lo que es importante un alto índice de sospecha en pacientes de riesgo para un diagnóstico precoz.
\end{abstract}

PALABRAS CLAVE: Cáncer colorrectal. Metástasis. Metástasis cutánea.

\begin{abstract}
Introduction: Colorectal cancer is a big health problem due to its high incidence and mortality. Cutaneous metastases are rare with an incidence $<5 \%$. Clinical case: $A$ male with a history of colectomy due to colon cancer, who 6 years later presented a cutaneous lesion. The biopsy reported colonic metastasis, so radical surgical resection was performed. The pathological anatomy confirmed a moderately differentiated adenocarcinoma compatible with colonic metastasis. Discussion and conclusion: Cutaneous metastases are rare and when they appear they indicate an advanced disease, for that reason is important a high index of suspicion in patients at risk, for an early diagnosis.
\end{abstract}

KEY WORDS: Colorectal cancer. Metastasis. Cutaneous metastasis.

\section{Correspondencia:}

*Jeancarlos J. Trujillo-Díaz

Av. Ramón y Cajal, 3

47003 Valladolid, España

E-mail: jeancar5671@gmail.com
Fecha de recepción: 21-02-2018

Fecha de aceptación: 21-04-2018

DOI: 10.24875/CIRU.M18000041
Cir Cir. 2018;86:285-288

Contents available at PubMed www.cirugiaycirujanos.com 


\section{Introducción}

El cáncer colorrectal constituye un gran problema de salud tanto por su alta incidencia como por su mortalidad. Según datos de la Organización Mundial de la Salud, el cáncer colorrectal es el tercer tumor más frecuente en hombres (10\% del total de cáncer mundial) y el segundo más frecuente en mujeres (9.2\% del total de cáncer mundial). En España, el cáncer colorrectal es actualmente el tumor maligno más frecuente.

El cáncer colorrectal tiene diseminación por vía linfática, peritoneal, hematógena y por contigüidad. El órgano que con mayor frecuencia se ve afectado en la diseminación hematógena es el hígado, estimándose que aproximadamente la mitad de los pacientes con cáncer colorrectal desarrollarán metástasis hepáticas en algún momento de la evolución de la enfermedad".

Por otra parte, las metástasis cutáneas del cáncer colorrectal son raras, con una incidencia menor del $5 \%$. En general, las metástasis cutáneas ocurren entre el 0.7 y el $5 \%$ de los pacientes con alguna neoplasia visceral, llegando a ocurrir hasta en un $10 \%$ en pacientes con cáncer metastásico².

Comentamos el caso de un paciente con neoplasia de colon que presenta una lesión cutánea única en la pared abdominal.

\section{Caso clínico}

Paciente varón de 86 años, con antecedentes médicos de hipertensión arterial, cardiopatía isquémica crónica, y quirúrgicos de nefrectomía izquierda por tumor renal (1998), prótesis de cadera izquierda, colectomía subtotal por neoplasia de colon sigmoide y poliposis adenomatosas (2012), metastasectomía hepática de lesión sincrónica en el segmento VIII (2012) y peritonitis por perforación intestinal (2013). Acude a control a la consulta y refiere presentar una lesión cutánea en el abdomen, 6 años después de la primera cirugía de neoplasia de colon. A la exploración se visualiza en la pared abdominal, en el flanco izquierdo, una lesión cutánea nodular, de forma ovalada, sobreelevada, hiperpigmentada, de $3 \times 2 \mathrm{~cm}$, blanda a la palpación y no dolorosa (Fig. 1). Se realiza analítica sanguínea con marcadores tumorales, los cuales no presentan alteraciones reseñables. En la última tomografía de control realizada no se observaban nuevas lesiones compatibles con recidiva.

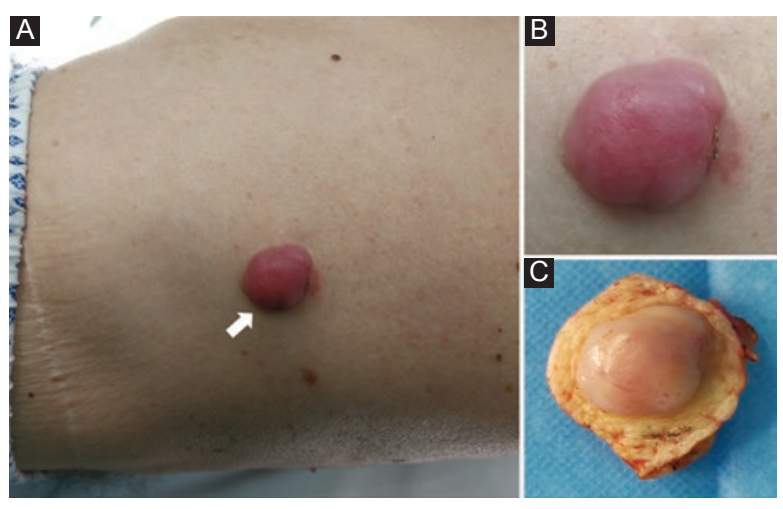

Figura 1. A: visión panorámica de la lesión cutánea localizada en la región lateral izquierda de la pared abdominal (flecha). B: lesión cutánea nodular, sobreelevada, hiperpigmentada, de $3 \times 2 \mathrm{~cm}$. C: pieza quirúrgica tras exéresis radical con márgenes libres de infiltración neoplásica.

Inicialmente se realiza biopsia de la lesión, que es informada como adenocarcinoma moderadamente diferenciado compatible con metástasis de origen colónico. Dado este hallazgo, se decide realizar exéresis quirúrgica radical de la lesión. La anatomía patológica de la pieza quirúrgica confirma que se trata de un adenocarcinoma moderadamente diferenciado compatible con metástasis de origen colónico. La tumoración se encuentra ocupando completamente la dermis y alcanza el tejido celular subcutáneo superficialmente; los márgenes quirúrgicos laterales y profundos se encontraban libres de infiltración neoplásica (Fig. 2).

\section{Discusión}

Las metástasis cutáneas del cáncer colorrectal suponen un $2 \%$ de todos los tumores de piel y con frecuencia aparecen junto con metástasis en otros órganos. Los cánceres que con mayor frecuencia metastatizan en la piel son el melanoma, el cáncer de mama y el cáncer de vías respiratorias altas, siendo el cáncer colorrectal un origen muy raro de metástasis cutáneas ${ }^{1}$. El hallazgo de metástasis cutáneas en el momento de la presentación del tumor primario se da en aproximadamente el $1.3 \%$ de los casos $^{3}$. Las metástasis cutáneas ocurren en aproximadamente el $5 \%$ de los cánceres colorrectales ${ }^{4}$.

Los sitios más frecuentes de metástasis cutánea de cáncer colorrectal son el abdomen (suelen localizarse en zonas próximas al tumor primario y se deben a extensión directa o implantación local durante la cirugía en la zona de la incisión quirúrgica), las extremidades, el periné, la cabeza, el cuello y el pene, ya sea por diseminación linfática o hematógena, o por implantación directa durante la cirugía ${ }^{3,4}$. 


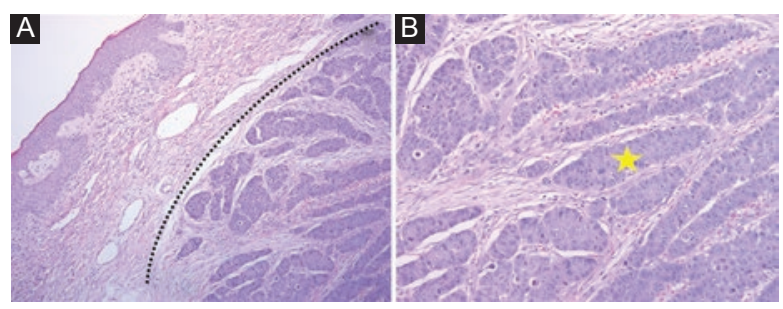

Figura 2. Hallazgos histológicos. A: visión a 10x: epidermis y dermis superficial libres de infiltración neoplásica (por encima de la línea de puntos). B: visión a 20x: intensa infiltración tumoral de la dermis reticular. Adenocarcinoma moderadamente diferenciado (estrella).

En un estudio realizado por $\mathrm{Hu}$, et al. ${ }^{5}$ se reportaron los orígenes primarios de las metástasis de la siguiente manera: recto $55 \%$, colon sigmoide $17 \%$, colon transverso $9 \%$, rectosigma $7 \%$, ciego $4 \%$ y colon ascendente $4 \% 5$.

Las metástasis cutáneas del cáncer colorrectal generalmente ocurren dentro de los primeros 2 años tras la resección del tumor colorrectal primario, y a menudo se presentan simultáneamente con metástasis en el hígado, el peritoneo y el pulmón ${ }^{3,6}$.

Las metástasis aparecen cuando las células neoplásicas se desprenden de un tumor primario y se diseminan a otros lugares. El mecanismo de producción de las metástasis cutáneas es variable y se han implicado varías vías en su desarrollo. Las vías hematógena y linfática son las más frecuentes, aunque la diferenciación entre ambas es difícil debido a que están interconectadas. La diseminación linfática es la vía más frecuente de propagación inicial de la mayoría de los tumores malignos. La implantación directa de células tumorales puede producirse por el transporte mecánico de fragmentos tumorales mediante los instrumentos quirúrgicos durante la cirugía u otros procedimientos invasivos, pero rara vez ocurre ${ }^{7}$.

Las presentaciones clínicas de las metástasis cutáneas son variadas e inespecíficas, siendo la forma más frecuente como nódulos dérmicos o subcutáneos de 1-2 cm de diámetro, indoloros, indurados, móviles, simples o múltiples, sin cambios en la epidermis suprayacente. En ocasiones pueden presentar un rápido crecimiento, bullas o procesos fibróticos, o ulcerarse y producir celulitis ${ }^{1,3}$.

Dada la rareza de estas lesiones y que no tienen un aspecto uniforme de presentación, no es raro que inicialmente se diagnostiquen de manera errónea como quistes simples o lesiones benignas del tejido conectivo, granulomas piógenos, tumores de células granulares, quistes benignos, neurofibromas o lipomas. Por ello, ante lesiones nodulares atípicas o persistentes en pacientes con antecedentes de neoplasias malignas hay que considerar realizar una biopsia para descartar la posibilidad de metástasis ${ }^{2,4}$.

Entonces, si sospechamos la afectación cutánea de una neoplasia maligna hay dos formas de obtener tejido para la confirmación diagnóstica: mediante biopsia o mediante punción-aspiración con aguja fina, pudiendo ser esta última más rápida y eficiente ${ }^{1}$. El diagnóstico se basa en los aspectos morfológicos e histológicos, y en la inmunohistoquímica, de la lesión cutánea, junto con la comparación de la morfología y la histología del tumor primario ${ }^{3}$. Las características morfohistológicas de las lesiones metastásicas en general se parecen a las del tumor primario. Sin embargo, con frecuencia las metástasis son más anaplásicas.

Las metástasis cutáneas pueden clasificarse en general como adenocarcinoma, carcinoma escamoso, carcinoma indiferenciado y otros tipos. La mayoría de las metástasis cutáneas de los tumores colorrectales están bien diferenciadas, y a menudo son adenocarcinomas secretores de mucina. Suelen tener una configuración nodular y se localizan en la dermis, con posterior diseminación a la epidermis y el tejido subcutáneo ${ }^{2}$. Cuando los tumores son poco diferenciados o anaplásicos, los estudios inmunohistoquímicos de detección, como CK7, CK20, CK19, CDX2, CEA y mucina, son de mucha utilidad ${ }^{4,6}$.

El tratamiento de las metástasis cutáneas del cáncer colorrectal es limitado y no existe una estrategia terapéutica estandarizada. Aunque el tratamiento del cáncer colorrectal metastásico se ha basado en la quimioterapia sistémica, la resección quirúrgica en pacientes seleccionados con metástasis cutáneas ofrece la única posibilidad de supervivencia a largo plazo ${ }^{3}$. Para lesiones aisladas, Nesseris, et al..$^{2}$ sugirieron una escisión local amplia con posterior reconstrucción. Sin embargo, Wong, et al. ${ }^{8}$ propusieron la resección típica con un margen de $1 \mathrm{~cm}$ de piel sana, y en algunas áreas de alta importancia estética o cuando el tratamiento sea paliativo pueden realizarse las extirpaciones con márgenes mucho más limitados.

Para los pacientes con metástasis cutáneas múltiples o lesiones irresecables puede considerarse la quimioterapia sistémica. Sin embargo, no existe un régimen de quimioterapia óptimo. Los tratamientos de quimioterapia establecidos incluyen 5-fluorouracilo (5-FU), capecitabina, irinotecán, oxaliplatino y cisplatino. Las combinaciones de irinotecán y 5-FU, oxaliplatino y 5-FU y leucovorina (LV) (FOLFOX), o 5-FU/LV con irinotecán (FOLFIRI), han aumentado la supervivencia a más de 20 meses. El tratamiento secuencial de los pacientes con FOLFIRI seguido de FOLFOX, o con FOLFOX 
seguido de FOLFIRI, ha aumentado la mediana de los tiempos de supervivencia a 21.5 meses y 20.6 meses, respectivamente $^{2}$. La radioterapia, la poliquimioterapia, la infusión aislada de las extremidades, las inyecciones de interferón alfa, la crioterapia, la ablación con láser, la ablación por radiofrecuencia, la crema de imiquimod al $5 \%$ y la terapia dirigida al oncogén también son tratamientos utilizados, pero sin evidencia científica que demuestre su eficacia ${ }^{3}$.

Por tanto, podemos decir que el hallazgo de una metástasis cutánea es un dato que indica que nos encontramos ante un estadio avanzado del cáncer colorrectal, con una esperanza de vida después del diagnóstico de las metástasis cutáneas de entre 1 y 34 meses, y por ende el tratamiento será generalmente paliativo y estará dirigido a mejorar la calidad de vida del paciente ${ }^{2}$. Lo más importante es hacer una detección y un tratamiento precoces del cáncer colorrectal para poder prevenir o disminuir la aparición de metástasis cutáneas diseminadas y aumentar así la esperanza de vida de estos pacientes ${ }^{6}$.

\section{Conclusiones}

En conclusión, las metástasis cutáneas del cáncer colorrectal presentan una baja incidencia, pero cuando aparecen generalmente indican una enfermedad avanzada y por tanto un mal pronóstico. El punto clave es el diagnóstico precoz, lo cual requiere una exploración física meticulosa y un alto índice de sospecha en pacientes de riesgo, ya que estas lesiones pueden pasar desapercibidas durante periodos prolongados. Ante la sospecha de metástasis cutáneas es esencial realizar una biopsia para confirmar el diagnóstico y así estadificar la enfermedad y plantearnos el mejor manejo terapéutico, pudiendo ser médico o quirúrgico.

\section{Conflicto de intereses}

No existen conflictos de intereses en ninguno de los autores.

\section{Bibliografía}

1. De Miguel M, Fraile M, Yagüe A, Oteiza F, Ciga M, Armendáriz $P$, et al. Metástasis cutáneas de cáncer de recto. An Sist Sanit Navar. 2013; 36:557-61.

2. Nesseris I, Tsamakis C, Gregoriou S, Ditsos I, Christofidou E, Rigopoulos D. Cutaneous metastasis of colon adenocarcinoma: case report and review of the literature. An Bras Dermatol. 2013;88:56-8.

3. Wang DY, Ye F, Lin JJ, Xu X. Cutaneous metastasis: a rare phenomenon of colorectal cancer. Ann Surg Treat Res. 2017;93:277-80.

4. Fragulidis GP, Vezakis A, Derpapas MK, Michalaki V, Tsagkas A, Polydorou AA. Cutaneous metastatic adenocarcinoma of the colon to the scalp. World J Oncol. 2015;6:304-7.

5. Hu S, Chen G, Lu Y, Wu C, Lan C. Cutaneous metastases from different internal malignancies: a clinical and prognostic appraisal. J Eur Acad Dermatol Venereol. 2008;22:735-40.

6. Hashimi Y, Dholakia S. Facial cutaneous metastasis of colorectal adenocarcinoma. BMJ Case Rep. 2013;2013:bcr2013009875.

7. Fernández-Antón M, Parra-Blanco V, Avilés J, Suárez R. Metástasis cutáneas de origen visceral. Actas Dermosifiliogr. 2013;104:841-53.

8. Wong CY, Helm MA, Kalb RE, Helm TN, Zeitouni NC. The presentation, pathology, and current management strategies of cutaneous metastasis. N Am J Med Sci. 2013:5:499-504. 